\title{
Factibilidad agronómica del uso de vinaza, subproducto de la fabricación del pisco, como biofertilizante en viñedos
}

\section{Agronomic feasibility of using vinasse, a by-product of the alcoholic spirit pisco, as a biofertilizer in vineyards}

RODRIGO CALLEJAS 1,2

ÁLVARO SILVA ${ }^{1}$

CECILIA PEPPI ${ }^{1}$

ÓSCAR SEGUEL'1

Viñedo Chardonnay, localidad de Punitaqui, Ovalle, Chile.

Foto: R. Callejas

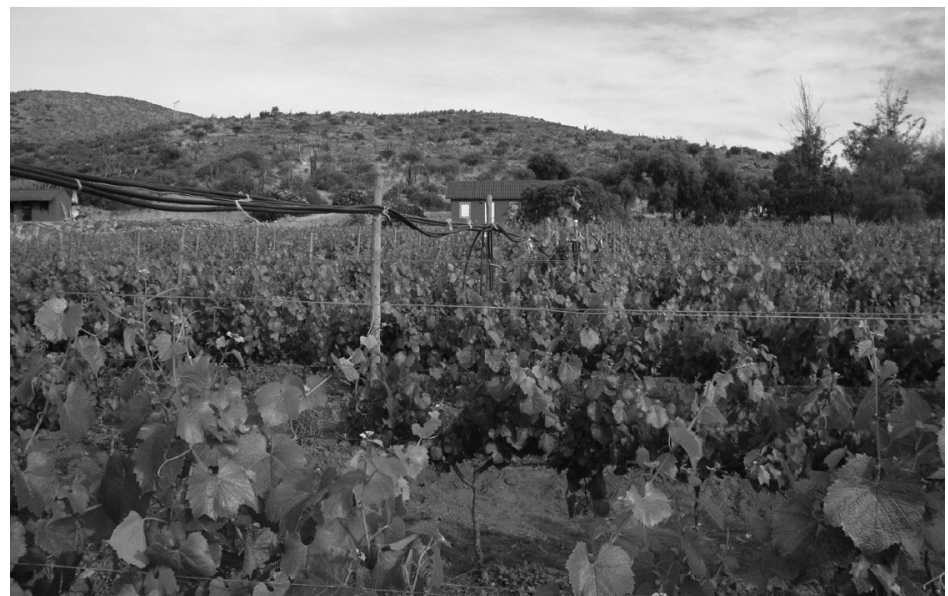

\section{RESUMEN}

La vinaza, subproducto orgánico líquido de la fabricación del pisco, es catalogada en Chile como desecho industrial, generándose la necesidad de lograr su reclasificación y promover su uso como biofertilizante. Para esto, se propusieron tres experimentos entre los años 2010 y 2012, con el objetivo de definir dosis que no provocaran efectos negativos en el sistema radical, el crecimiento aéreo de las vides y características químicas del suelo. En los dos primeros experimentos se aplicó el equivalente a 0, 168, 336 y $672 \mathrm{~m}^{3}$ ha $^{-1}$ en la var. Pedro Jiménez, utilizando un rizotrón estrella de un 10,1 L de cuatro puntas (experimento uno) y maceta de $20 \mathrm{~L}$ (experimento dos). El tercer experimento se realizó en campo, var. Chardonnay, con cuatro tratamientos (0, 103, 206 y $412 \mathrm{~m}^{3} \mathrm{ha}^{-1}$ ) utilizando grupos de cuatro plantas como unidad experimental. En los dos primeros experimentos se definió que la dosis de $336 \mathrm{~m}^{3}$ ha-1 no afectaban la parte aérea y promovía mayor crecimiento del sistema radical. En el experimento tres, cantidades de hasta $206 \mathrm{~m}^{3} \mathrm{ha}^{-1}$ no causaron cambios químicos a nivel de suelo o la parte aérea de la planta, pero promovieron un mayor índice de enraizamiento en los primeros $20 \mathrm{~cm}$. El uso adecuado de la vinaza de pisco podría transformarse en una buena oportunidad para los pequeños agricultores, al utilizarse como enmienda orgánica o biofertilizante agrícola en viñedos.

Palabras clave adicionales: Vitis vinifera, raíz, residuos agroindustriales, enmienda orgánica.

Facultad de Ciencias Agronómicas, Universidad de Chile, Santiago (Chile).

Autor para correspondencia: rcalleja@uchile.cl; rcallejas.uchilecrea@gmail.com 


\section{ABSTRACT}

Vinasse is an organic, liquid by-product of pisco alcoholic spirit manufacturing, categorized in Chile as industrial waste, resulting in a need for re-classification and promotion of its use as a biofertilizer. Three experiments were carried out between 2010 and 2012 in order to determine the doses that do not negatively affect grapevine root systems, aboveground growth, or soil chemical variables. Experiments 1 and $2(0,168$, 336 and $672 \mathrm{~m}^{3} \mathrm{ha}^{-1}$ ) were conducted with $\mathrm{cv}$. Pedro Jiménez. The experimental unit consisted of one fourtipped, star-shaped 10.1 L rhizotron (experiment 1) or one plant in a $20 \mathrm{~L}$ container (experiment 2). The third experiment was established in the field using cv. Chardonnay with 4 treatments $\left(0,103,206\right.$ and $\left.412 \mathrm{~m}^{3} \mathrm{ha}^{-1}\right)$ and an experimental unit that corresponded to a group of 4 plants. In the first two experiments, a dose of $336 \mathrm{~m}^{3} \mathrm{ha}^{-1}$ did not affect the aboveground growth but promoted root development. In experiment 3 , doses of up to $206 \mathrm{~m}^{3}$ ha-1 did not change the chemical composition of the soil or the aboveground growth, but stimulated a higher rooting index in the first $20 \mathrm{~cm}$ of the soil profile. The appropriate use of vinasse, a byproduct of pisco, currently classified as an industrial waste, could provide a good alternative for grape growers when utilized as an organic amendment or biofertilizer in vineyards.

Additional key words: Vitis vinifera, root, agroindustrial waste, organic amendment.

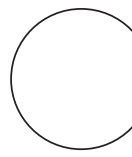

La vinaza en Chile es un subproducto orgánico líquido de la fabricación de pisco, industria por denominación de origen circunscrita a las regiones de Atacama y Coquimbo y de cuya destilación del vino se genera $25 \%$ de alcohol y $75 \%$ de vinaza (CPL, 2004). La vinaza es considera$\mathrm{da}$ indeseable debido a los efectos secundarios con los que se asocia, como la contaminación de ríos, fuentes de aguas subterráneas y mares, generando muerte de peces y un grave problema ambiental (Pérez y Garrido, 2008). Este producto se considera un residuo industrial líquido (RIL), con un costo de tratamiento y eliminación de US\$ 300.000 por año para una planta que genera $200 \mathrm{~m}^{3} \mathrm{~d}^{-1}$ de vinaza. Su alto déficit biológico de oxígeno (DBO5) dificulta su uso, pues la Normativa Chilena 1.333 de Calidad de Aguas de Riego permite aplicar cantidades máximas de vinaza en lugares especialmente habilitados y controlados, equivalentes a 112 kg DBO5 ha-1 d-1 (SAG, 2006). Según Alfaro y Alfaro (1996), con un buen manejo la vinaza se podría utilizar, permitiendo beneficiarse de la alta concentración de $\mathrm{K}$ (732 $\left.\mathrm{mg} \mathrm{L}^{-1}\right)$, que es esencial para los cultivos. Singh et al. (1998), aplicaron vinaza a varios niveles de DBO5 (0 a $3.000 \mathrm{mg} \mathrm{L}^{-1}$ ) y encontraron que $500 \mathrm{mg}$ DBO5 $\mathrm{L}^{-1}$ permitieron cultivar trigo (Triticum aestivum) durante 3 años sin problemas; además, el $\mathrm{pH}$ y la CE del suelo no variaron, aumentando el contenido de C, N, P y K. Mora et al. (2010) observaron que $125 \mathrm{~m}^{3} \mathrm{ha}^{-1}$ de vinaza incrementaron el rendimiento de papa (Solanum tuberosum), de 33,48 a 44,23 $\mathrm{t} \mathrm{ha}^{-1}$, superando a la fertilización química (150-250-60-30 kg ha-1 de $\mathrm{N}^{-} \mathrm{P}_{2} \mathrm{O}_{5}$ $\mathrm{K}_{2} \mathrm{O}-\mathrm{S}$ ), con la que se alcanzaron $37,41 \mathrm{t} \mathrm{ha}^{-1}$. Armengol et al. (2003) usaron $150 \mathrm{~m}^{3} \mathrm{ha}^{-1} \mathrm{de}$ vinaza en caña de azúcar (Saccharum officinarum) y observaron un rendimiento mayor $(130,85 \mathrm{t}$ $\left.\mathrm{ha}^{-1}\right)$ que la fertilización mineral $\left(95,27 \mathrm{t} \mathrm{ha}^{-1}\right)$, a valores de 60,2 $\mathrm{kg} \mathrm{N}^{-1}, 25 \mathrm{~kg} \mathrm{P}_{2} \mathrm{O}_{5} \mathrm{ha}^{-1} \mathrm{y}$ $80 \mathrm{~kg} \mathrm{~K}_{2} \mathrm{O} \mathrm{ha}^{-1}$. La vinaza en dosis moderadas $\left(150 \mathrm{~m}^{3} \mathrm{ha}^{-1}\right)$ satisface las necesidades de $\mathrm{N} \mathrm{y}$ K en caña de azúcar (Korndorfer et al., 2010) y su efecto como abono orgánico es mejorar las características físicas, químicas y biológicas del 
suelo, aumentar la fertilidad y reducir la aplicación de fertilizantes sintéticos (Urbano, 2002). En este sentido, Valverde et al. (2004) señalan que es un producto completo, pues aporta los nutrientes necesarios para las plantas y mejora las condiciones físicas, químicas y bacteriológicas del suelo.

Dado que no hay antecedentes sobre el uso de esta vinaza en vides y existe el desafío de proponer su utilización como enmienda orgánica o biofertilizante bajo la normativa chilena, se planteó la tarea de determinar dosis que no provocaran efectos negativos, definiéndose los siguientes objetivos: 1) evaluar el efecto de la vinaza sobre plantas de vid, sistema radical y follaje, en ensayos en macetas y 2) determinar el efecto de la vinaza en condiciones de campo, sobre las propiedades químicas del suelo, la planta de vid y su producción.

\section{MATERIALES Y MÉTODOS}

Durante el 2010 y 2011, se realizaron dos experimentos en condiciones controladas en rizotrón y en macetas, en dependencias de la Facultad de Ciencias Agronómicas de la Universidad de Chile, Región Metropolitana (3334'12" S, 70³8'4" W, a $624 \mathrm{msnm}$ ), con clima templado cálido de veranos secos según la clasificación de Köppen (Villate, 2007). Un tercer experimento (años 2011 y 2012) a nivel de campo, se realizó en Punitaqui, IV Región de Coquimbo $\left(30^{\circ} 54^{\prime} 0^{\prime \prime} \mathrm{S}, 71^{\circ} 16^{\prime} 0^{\prime \prime} \mathrm{W}\right)$, Chile, a $450 \mathrm{msnm}$ de altitud, con clima semiárido de veranos secos según la clasificación de Köppen y precipitación anual promedio de $130 \mathrm{~mm}$. El análisis de la vinaza de los dos primeros experimentos presentó un pH de 3,6; CE de 3,7 dS m-1; 0,89\% de carbono orgánico; $0,06 \mathrm{mg} \mathrm{L}^{-1}$ de $\mathrm{N}$ total; 64 $\mathrm{mg} \mathrm{L}^{-1}$ de $\mathrm{P}$ total; $732 \mathrm{mg} \mathrm{L}^{-1}$ de $\mathrm{K}$ total y una relación $\mathrm{C} / \mathrm{N}$ de 14,8 . No se realizó ningún tipo de fertilización mineral. Para el tercer experimento, la vinaza presentó un pH de 3,6; CE de $3,9 \mathrm{dS} \mathrm{m}^{-1} ; 1,0 \%$ de carbono orgánico; $0,08 \%$ de
$\mathrm{N}$ total; $62 \mathrm{mg} \mathrm{L}^{-1}$ de $\mathrm{P}$ total; $924 \mathrm{mg} \mathrm{L}^{-1}$ de $\mathrm{K}$ total y una relación $\mathrm{C} / \mathrm{N}$ de 13,5 .

\section{Primer experimento}

Se estableció en noviembre del 2010, utilizando un rizotrón tipo estrella (Wright y Wright, 2004; Silva y Beeson, 2011) de cuatro extremos y 10,1 L cada uno. Cada rizotrón estuvo compuesto por ocho caras de $25 \times 40 \mathrm{~cm}$ unidas en los extremos exteriores por caras de 10x25 cm. Se establecieron plantas de Vitis vinifera variedad Pedro Jiménez, francas, de un año de edad, usando un sustrato con $50 \%$ arena y $50 \%$ suelo franco, con un contenido de 1,9\% $\mathrm{MO}, 40 \mathrm{mg}$ $\mathrm{L}^{-1}$ de N, $14 \mathrm{mg} \mathrm{L}^{-1}$ de P y $92 \mathrm{mg} \mathrm{L}^{-1}$ de K. Se realizaron cuatro tratamientos de vinaza equivalentes a $0,168,336$ y $672 \mathrm{~m}^{3} \mathrm{ha}^{-1}$ subdivididas en 20 aplicaciones (de fines de noviembre a mediados de enero), regándose cada $2 \mathrm{~d}$ con un volumen total de $674 \mathrm{~cm}^{3}$ de solución por cuadrante y controlado con un equipo TDR100® (Spectrum Technologies, Plainfield, IL). Solamente para la dosis mayor (672 $\left.\mathrm{m}^{3} \mathrm{ha}^{-1}\right)$ fue necesario implementar un lavado extra cada tres riegos con $1.250 \mathrm{~m}^{3}$ de agua para evitar la acumulación excesiva de sales, dado que con valores mayores a un $30 \%$ de vinaza en la solución utilizada para regar, la CE se incrementaba por sobre $1,70 \mathrm{dS} \mathrm{m} \mathrm{m}^{-1}$. En las secciones testigo se regó solo con agua. El diseño experimental fue de bloques completamente aleatorizado, con ocho repeticiones correspondientes a una planta en la cual cada punta o sección consistió en un tratamiento. Transcurridos 100 d desde el inicio del experimento, se evaluó peso fresco de raíces, largo y diámetro del sector medio de raíces agrupadas según el peso fresco (Van Zyl, 1988). Para obtener el peso seco de raíces, la masa radical se mantuvo en una estufa de secado a $60^{\circ} \mathrm{C}$ hasta alcanzar peso constante.

\section{Segundo experimento}

En foma paralela, se estableció el mismo tipo de plantas de vid en macetas individuales de 
$20 \mathrm{~L}$, con el mismo sustrato y tratamientos del experimento 1, subdivididas en 10 aplicaciones (de fines de noviembre a mediados de diciembre), regándose cada $2 \mathrm{~d}$ con un volumen total de $1.455 \mathrm{~cm}^{3}$ de solución por maceta. En la dosis mayor se implementó un lavado extra cada tres riegos con $7.000 \mathrm{~cm}^{3}$ de agua por maceta para evitar la acumulación excesiva de sales. Se usaron 32 macetas individuales, el diseño experimental fue bloques completamente aleatorizado con ocho repeticiones, utilizando la planta como la unidad experimental. Se realizaron las mismas evaluaciones que en el experimento anterior, agregando el peso fresco y seco aéreo.

\section{Tercer experimento}

Se inició en agosto del 2011 y terminó en junio de 2012. Se usaron vides de 4 años de edad, var. Chardonnay, plantadas a 2,5x1,0 m con riego por goteo con doble línea. El suelo presenta una textura franco arcillo arenosa, moderadamente profundo y con una pendiente de 1 a $3 \%$. El diseño experimental fue en bloques completamente aleatorizados, con cuatro tratamientos de vinaza: 0, 103, 206 y $412 \mathrm{~m}^{3} \mathrm{ha}^{-1}$, equivalentes a 0 , 25,8; 51,5 y $103 \mathrm{~L}$ por planta, respectivamente, con cinco repeticiones, siendo la unidad experimental un grupo de cuatro plantas. Las dosis fueron menores a los ensayos en contenedores para ajustarse a las exigencias medio ambientales (SAG, 2006). La fertilización del testigo fue de $40 \mathrm{~kg} \mathrm{ha}^{-1} \mathrm{~N}$ como urea y $30 \mathrm{~kg} \mathrm{ha}^{-1} \mathrm{~K}$ como sulfato de potasio; para $103 \mathrm{~m}^{3} \mathrm{ha}^{-1}, 30,9 \mathrm{~kg} \mathrm{ha}^{-1}$ $\mathrm{N}$ como urea y $9,1 \mathrm{~kg} \mathrm{ha}^{-1} \mathrm{~N}$ de la vinaza; para $206 \mathrm{~m}^{3} \mathrm{ha}^{-1}, 21,7 \mathrm{~kg} \mathrm{ha}^{-1} \mathrm{~N}$ como urea y $18,3 \mathrm{~kg}$ $\mathrm{ha}^{-1} \mathrm{~N}$ de la vinaza; y para $412 \mathrm{~m}^{3} \mathrm{ha}^{-1}, 3,4 \mathrm{~kg}$ ha ${ }^{-1} \mathrm{~N}$ como urea y $36,6 \mathrm{~kg} \mathrm{ha}^{-1} \mathrm{~N}$ de la vinaza. A las plantas con vinaza no se requirió aplicar $\mathrm{K}$. La vinaza fue incorporada en cada riego en concentraciones de $0 ; 2,5 ; 5$ y $10 \%$ desde baya de $3 \mathrm{~mm}$, finalizando con el último riego de la temporada. Para evitar la percolación profunda exigida por la autoridad medioambiental por efecto del riego (SAG, 2006), el contenido de agua del suelo se controló con una sonda electromag- nética de lectura continua marca Enviroscan (Sentek), con cuatro sensores (a 15, 25, 45 y 85 $\mathrm{cm}$ de profundidad) que permitieron definir el tiempo y frecuencia de riego. La retención de agua por horizonte genético se determinó a tensiones de 33 y $1.500 \mathrm{kPa}$ (capacidad de campo y punto de marchitez permanente). La demanda hídrica hasta el 26 de abril alcanzó un volumen equivalente a $4.000 \mathrm{~m}^{3} \mathrm{ha}^{-1}$ y se realizaron 25 riegos con vinaza. El análisis foliar (lámina) de $\mathrm{N}$ (Kjeldahl), P (fotocolorimétrico), K, Mg y Ca (espectrofotometría de absorción atómica) se realizó en envero y cosecha de acuerdo a Cadahía (2005). Se registró la producción por planta, peso de racimos, número de racimos, contenido de sólidos solubles a la cosecha (entre el 19 y 24 de febrero del 2012) y el peso de poda. Al final del ensayo, se evaluó el contenido de N, P, K, $\mathrm{MO}$ y valores de $\mathrm{CE}$ y pH en el suelo (Sadawka et al. 2006) a profundidades de 5-20, 20-35, 3550 y $70-90 \mathrm{~cm}$, considerando tres repeticiones por tratamiento. En tres de las repeticiones se evaluó el número de raíces en las profundidades 0-20, 20-40 y 40-60 cm, siguiendo la metodología del mapeo detallado del perfil propuesto por Böhm (1979). Para esto se realizaron calicatas de $1 \mathrm{~m}$ de profundidad por $1 \mathrm{~m}$ de ancho, paralelas a la hilera y a $20 \mathrm{~cm}$ del tronco de las plantas, instalando una malla cuadriculada de 20 × 20 $\mathrm{cm}$, registrándose el número de raíces de cada una. Las raíces se clasificaron como en los experimentos anteriores y se determinó el índice de enraizamiento (IE) (Van Zyl, 1988), siguiendo la fórmula:

$$
\begin{array}{r}
\mathrm{IE}=[\mathrm{R}(<0,5 \mathrm{~mm})+\mathrm{R}(0,5-2 \mathrm{~mm})] \\
/[\mathrm{R}(2-5 \mathrm{~mm})+\mathrm{R}(>5 \mathrm{~mm})]
\end{array}
$$

donde $\mathrm{R}$ es el número de raíces en las diferentes categorías.

Para cada experimento, lo resultados fueron sometios a análisis de varianza y en el caso de existir diferencias, la separación de las medias se realizó con la prueba de Tukey $(P \leq 0,05)$ utilizando el programa Minitab Release. 


\section{RESULTADOS Y DISCUSIÓN}

\section{Experimentos 1 y 2}

En el experimento con vides en rizotrón, si bien las dos dosis superiores mostraron diferencias significativas de peso fresco comparado con el testigo, fue el tratamiento de $336 \mathrm{~m}^{3} \mathrm{ha}^{-1}$ el de mayor peso seco de raíces (figura 1A), situación generada principalmente por la mayor cantidad de raíces finas y medias (figura 1B). La dosis más alta $\left(672 \mathrm{~m}^{3} \mathrm{ha}^{-1}\right)$ generó valores que comenzaron a decaer y a una condición de crecimiento similar al testigo, estimándose que probablemente alguna característica de la vinaza, cuando es utilizada en vid en cantidades altas o en exceso, se volvería contraproducente para el óptimo desarrollo de las raíces.

En el segundo experimento, la información de las vides en macetas individuales ratifica los resultados del experimento con rizotrones, pues claramente la dosis mayor, equivalente a $672 \mathrm{~m}^{3} \mathrm{ha}^{-1}$, tiende a deprimir el crecimiento de raíces en relación al tratamiento anterior (336 $\mathrm{m}^{3} \mathrm{ha}^{-1}$ ), presentando un comportamiento similar al testigo (figura 2A). En este caso deprimió adicionalmente el crecimiento de la parte aérea (figura $2 \mathrm{~B}$ ), evaluado como peso fresco, observándose en las hojas basales una tenue amarillez. Estos experimentos muestran que dosis de $336 \mathrm{~m}^{3}$ ha-1 no generan efectos colaterales negativos que puedan indicar que el uso de la vinaza es contraproducente para las vides y menos en plantas adultas, promoviendo claramente un mayor tamaño del sistema radical y raíces más hidratadas que el testigo. Esta información se suma a los resultados obtenidos en brócoli (Brassica oleracea), maíz (Zea mays) y fríjol (Phaseolus vulgaris) (Valverde et al., 2004) y en pepino dulce (Solanum muricatum) (Santos et al., 2007), donde se ha observado una promoción del crecimiento de raíces, generando un mayor crecimiento de los cultivos.

Según Honorato et al. (1990) y Callejas et al. (2012), la calidad del sistema radical define en gran medida el desarrollo y vigor de las vides,

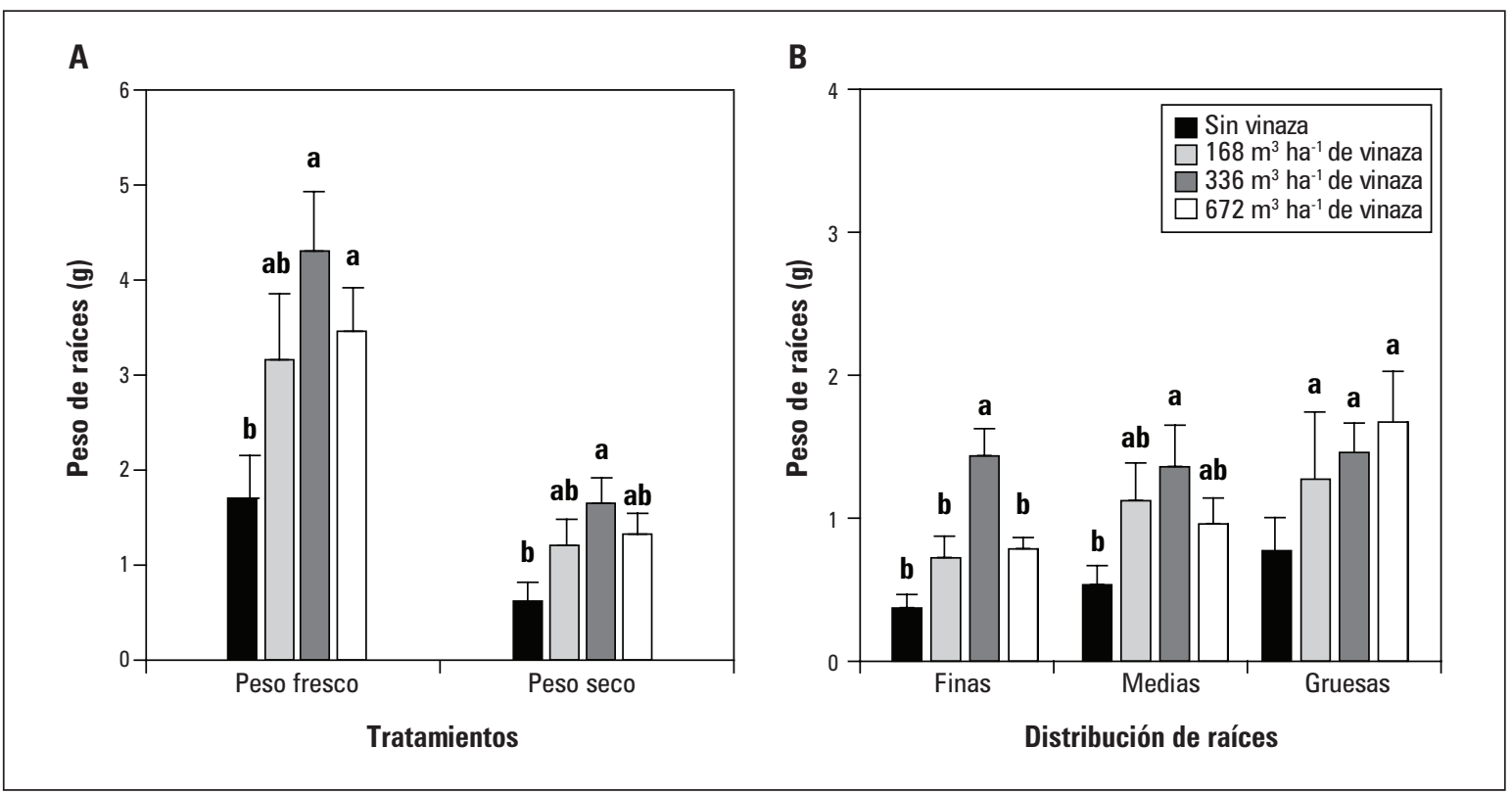

Figura 1. A. Peso fresco y seco de raíces y B. Distribución de acuerdo con su tamaño para diferentes tratamientos de vinaza en vides en rizotrón. Las barras indican el error estándar. Promedios con letras distintas indican diferencia significativa según la prueba de Tukey $(P \leq 0,05)$. 

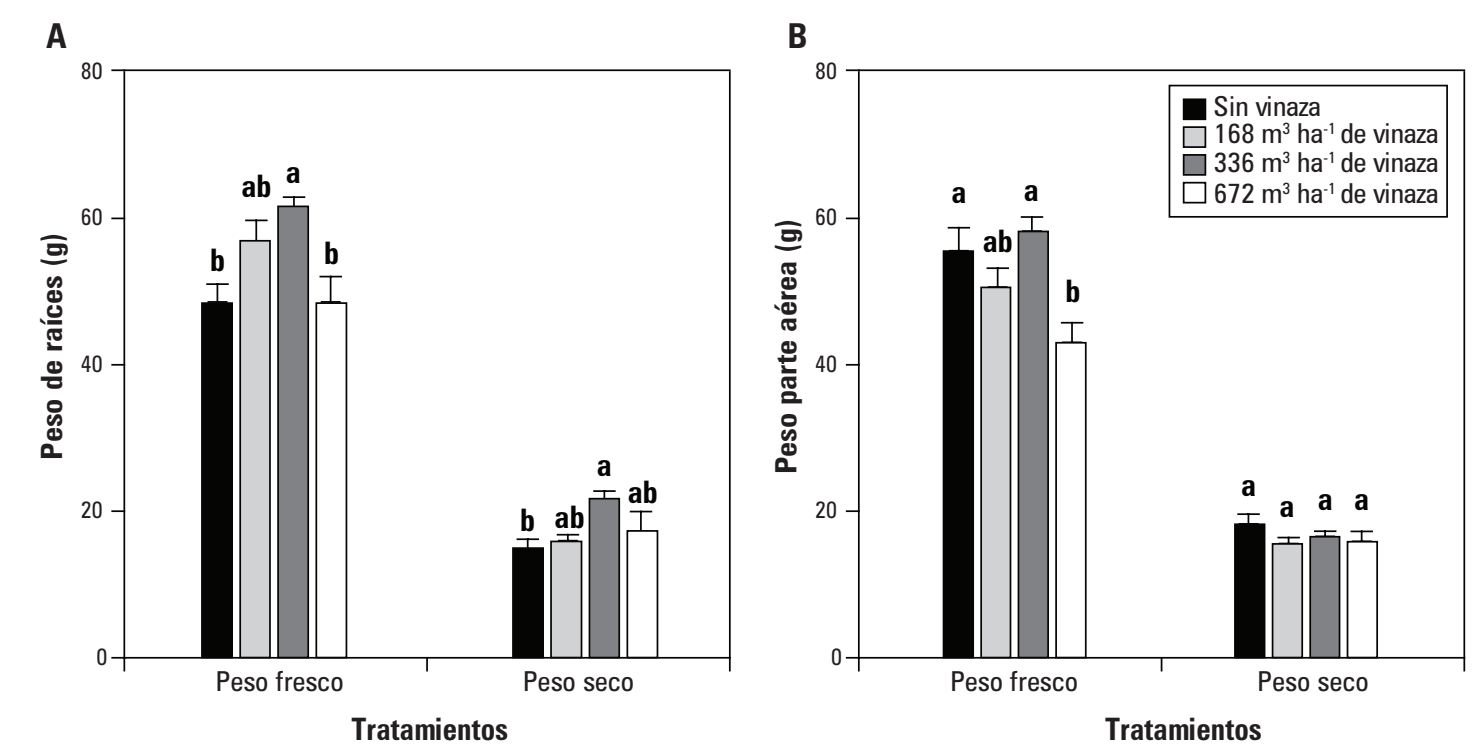

Figura 2. A. Peso fresco y seco de raíces y B. Peso fresco y seco de parte aérea de vid para diferentes tratamientos de vinaza. Las barras indican el error estándar. Promedios con letras distintas indican diferencia significativa según la prueba de Tukey $(P \leq 0,05)$.

así como los niveles productivos de los huertos, sobre todo en las condiciones de temperatura de suelo del desierto de Atacama (Callejas et al., 2009), cuando existe una adecuada disponibilidad hídrica. Callejas et al. (2012) ratifican para vides lo señalado por Sellés et al. (2003), en el sentido que desde el punto de vista de funcionalidad y actividad, las plantas que presentan una tendencia a ser más productivas poseen raíces de menor diámetro y de color blanco, dado que las raíces menores a $2 \mathrm{~mm}$ de diámetro son las más importantes en términos de la absorción de agua y nutrientes (Mullins et al., 1992). Por tanto, el buen manejo de los factores productivos impactará directamente en la calidad del sistema radical y con ello en el potencial productivo de las plantas (Glenn, 2000). Respecto al menor crecimiento observado con dosis altas de vinaza $\left(672 \mathrm{~m}^{3} \mathrm{ha}^{-1}\right)$ y atendiendo los cambios de la coloración de las hojas, habría que determinar en el futuro si la alta acumulación de $\mathrm{K}$ en la maceta pudo haber afectado la disponibilidad de $\mathrm{Mg}^{2+} \mathrm{o} \mathrm{Ca}^{2+}$. Otro factor que puede explicar el menor crecimiento sería la mayor presencia de sales en el suelo, a pesar del lavado incorporado en este tratamiento, con un efecto osmótico o por toxicidad de iones específicos (Ferguson y Grattan, 2005).

Finalmente, es posible que los niveles de $\mathrm{DBO} 5$ generados por esta dosis hayan disminuido los niveles de oxígeno en el suelo, afectando el crecimiento del sistema radical. En esta misma línea, Bengough et al. (2006) señalan que un simple estrés o la combinación de varios de ellos debido a condiciones del suelo, pueden limitar la elongación de las raíces, por lo que se debe evitar caer en excesos o promover deficiencias nutricionales y controlar otros aspectos como la salinidad.

\section{Experimento 3}

De acuerdo con la figura 3 (parte superior) y lo señalado por Obreza y Shumann (2010), no se produjo percolación profunda (sensor más profundo, $85 \mathrm{~cm}$ ), el cual determinó el tiempo de riego. Se observa que la mayor actividad del sis- 
tema radical se encuentra en los primeros horizontes del suelo (sensores a los 15 y $25 \mathrm{~cm}$ ), dado que el contenido de agua, de acuerdo con el sensor ubicado a $45 \mathrm{~cm}$, se mantiene estable en el tiempo, no habiendo raíces activas en profundidad. Finalmente, las condiciones hídricas del suelo fueron óptimas para que se expresaran los efectos de los tratamiento de vinaza (figura 3 , parte inferior, frecuencia de riego), debido a la clara dinámica radical (consumo de agua) en respuesta a la demanda atmosférica diaria.

No se detectaron diferencias significativas en el contenido de nutrientes en hojas (tabla 1). El N,
Ca y $\mathrm{Mg}$ se ubicaron dentro del intervalo adecuado para las vides (Christensen, 1984), mientras que el $\mathrm{K}$ se presentó ligeramente bajo y el P ligeramente alto. La falta de diferencias significativas, como señal de deficiencia o toxicidad inducidos por los tratamientos de vinaza, coincide con los resultados en otros cultivos obtenidos por Paula et al. (1999) en caña de azúcar (Saccharum officinarum) con dosis de 0 a $400 \mathrm{~m}^{3} \mathrm{ha}^{-1}$ de vinaza, y Paula et al. (1992) en cebolla (Allium cepa) con ocho dosis de vinaza ( 0 a $\left.210 \mathrm{~m}^{3} \mathrm{ha}^{-1}\right)$.

La fecha de cosecha, determinada como el punto en que la uva acumuló $22{ }^{\circ}$ Brix, fue similar

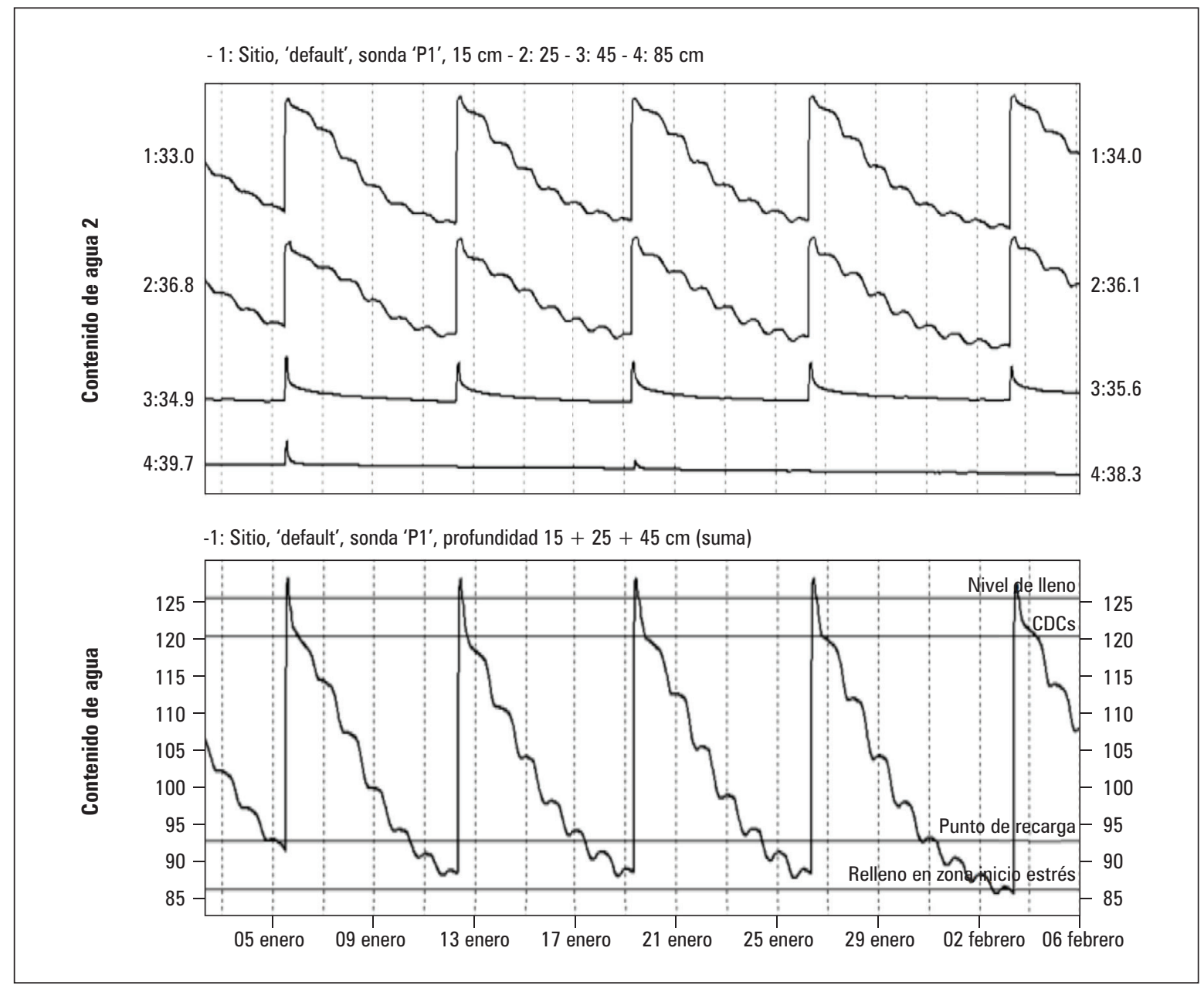

Figura 3. Comportamiento del contenido de agua del suelo a cuatro profundidades $(15,25,45$ y $85 \mathrm{~cm})$ evaluada con sonda electromagnética Enviroscan. Parte superior, actividad de las raíces por profundidad y control del tiempo de riego. Parte inferior, control de la frecuencia de riego. 
para todos los tratamientos. No se detectaron diferencias en producción (promedio $0,81 \mathrm{~kg} /$ planta), número de racimos ( $8,3 /$ planta) y peso de los racimos (118 g/racimo), coincidiendo con lo obtenido en caña de azúcar (Alfaro y Alfaro, 1996; Hernández et al., 2008). La mayor proporción de raíces estuvo en los primeros $40 \mathrm{~cm}$ de profundidad (figura 4A), concordando con la figura 3, parte superior, y lo señalado por Van
Zyl (1988), presentando un índice de enraizamiento medio comparado con parrones adultos de vid de mesa 'Thompson Seedless' (Callejas et al., 2012), probablemente por el menor desarrollo del sistema radical de vides sometidas a la producción pisquera. Queda claro que dosis de 103 y $206 \mathrm{~m}^{3} \mathrm{ha}^{-1}$ de vinaza promueven una mayor proporción de raíces finas en los primeros $20 \mathrm{~cm}$ del perfil, con diferencias estadísticas

Tabla 1. Concentración foliar de N, P, K, Ca y Mg en envero (E) y cosecha (C) en plantas de vid 'Chardonnay' con distintos tratamientos de aplicación de vinaza.

\begin{tabular}{|c|c|c|c|c|c|c|c|c|c|c|}
\hline \multirow{3}{*}{$\begin{array}{l}\text { Tratamientos } \\
\left(\mathrm{m}^{3} \mathrm{ha}^{-1}\right)\end{array}$} & \multicolumn{10}{|c|}{ Concentración foliar del elemento (\%) } \\
\hline & \multicolumn{2}{|c|}{$\mathrm{N}$} & \multicolumn{2}{|c|}{$\mathrm{P}$} & \multicolumn{2}{|c|}{ K } & \multicolumn{2}{|c|}{$\mathrm{Ca}$} & \multicolumn{2}{|c|}{$\mathrm{Mg}$} \\
\hline & $\mathrm{E}$ & C & $\mathrm{E}$ & $C$ & $\mathrm{E}$ & C & $\mathrm{E}$ & C & $\mathrm{E}$ & C \\
\hline 0 & $2,59 \mathrm{a}$ & $2,55 \mathrm{a}$ & $0,30 \mathrm{a}$ & $0,27 a b$ & $1,00 \mathrm{a}$ & $1,00 \mathrm{a}$ & $2,27 \mathrm{a}$ & $2,02 \mathrm{a}$ & $0,35 a$ & $0,33 \mathrm{a}$ \\
\hline 103 & $2,36 \mathrm{a}$ & $2,23 \mathrm{a}$ & $0,32 \mathrm{a}$ & $0,33 \mathrm{a}$ & $0,99 \mathrm{a}$ & $1,07 \mathrm{a}$ & $2,33 \mathrm{a}$ & $2,17 \mathrm{a}$ & $0,35 \mathrm{a}$ & $0,33 \mathrm{a}$ \\
\hline 206 & $2,55 \mathrm{a}$ & $2,15 \mathrm{a}$ & $0,30 \mathrm{a}$ & $0,29 a b$ & $0,86 \mathrm{a}$ & $0,97 \mathrm{a}$ & $2,42 \mathrm{a}$ & $2,32 \mathrm{a}$ & $0,36 \mathrm{a}$ & $0,37 \mathrm{a}$ \\
\hline 412 & $2,50 \mathrm{a}$ & $2,13 \mathrm{a}$ & $0,27 \mathrm{a}$ & $0,26 \mathrm{~b}$ & $0,92 \mathrm{a}$ & $0,94 \mathrm{a}$ & $2,40 \mathrm{a}$ & $2,15 \mathrm{a}$ & $0,36 \mathrm{a}$ & $0.33 \mathrm{a}$ \\
\hline
\end{tabular}

Promedios con letras distintas indican diferencia significativa según la prueba de Tukey $(P \leq 0,05)$.
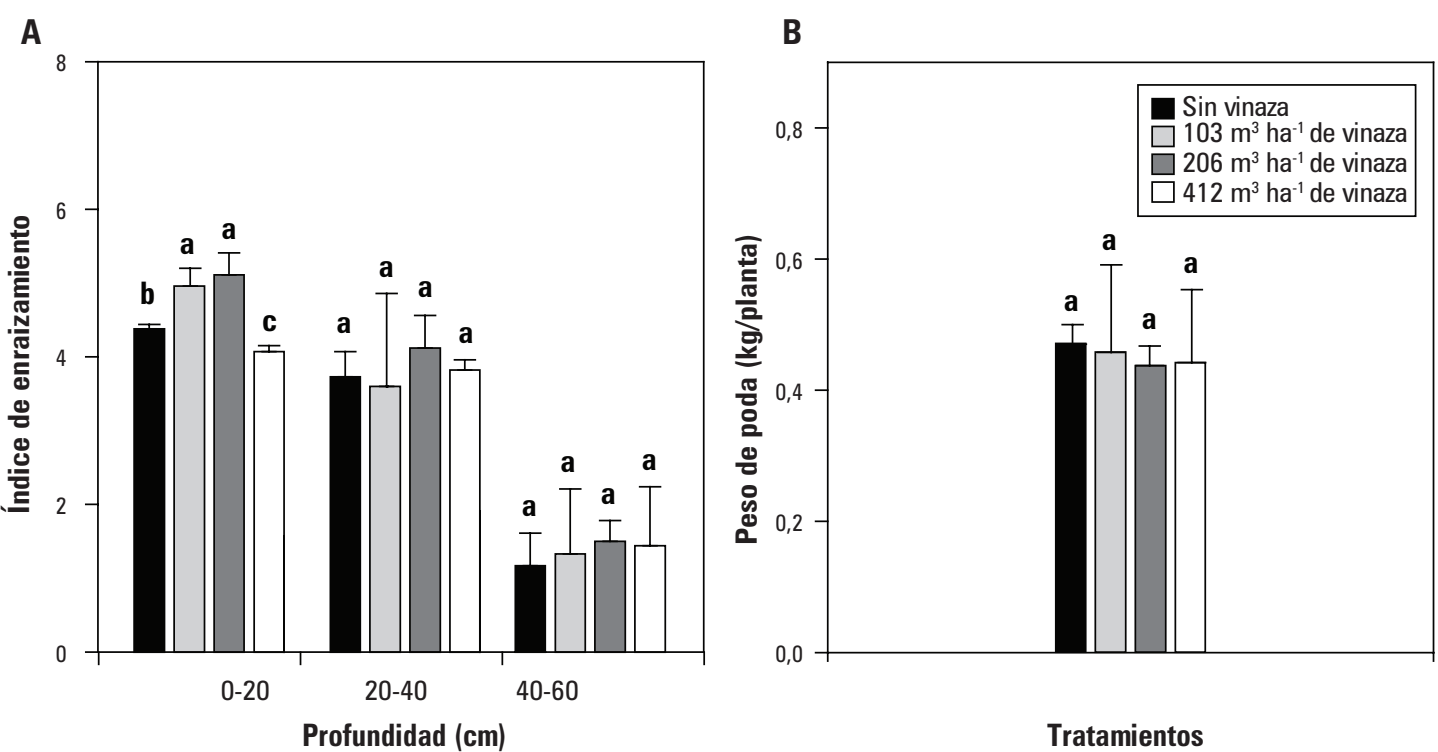

Figura 4. A. Índice de enraizamiento en función de la profundidad y B. Peso de la poda (kg por planta) de acuerdo con los diferentes tratamientos de vinaza. Las barras indican el error estándar. Promedios con letras distintas indican diferencia significativa según la prueba de Tukey $(P \leq 0,05)$. 
significativas en relación al testigo y a la dosis máxima. Sin embargo, esta mayor actividad de raíces no se tradujo en diferencias en el peso de poda (figura 4B), lo que permite concluir que el reemplazo de parte del $\mathrm{N}$ por los nutrientes contenidos en la vinaza permiten a la planta de vid lograr un equilibrio funcional, con un estado nutricional a cosecha similar al testigo con fertilización mineral.

Las propiedades relacionadas con la fertilidad del suelo se presentan en la figura 5, no observándose alteraciones indeseadas en el perfil que puedan ser una respuesta a la aplicación creciente de vinaza en el suelo. Para los cuatro tratamientos, según Callejas et al. (2011), el contenido de $\mathrm{N}$ en el suelo se encuentra en un nivel medio (figura $5 \mathrm{~A}$ ), el $\mathrm{K}$ en superficie es muy alto y medio en profundidad (figura 5C) y el $\mathrm{P}$ muy alto en superficie y va disminuyendo en profundidad (figura 5B). La CE se presentó en intervalos no salinos (figura 5D) sin un comportamiento definido y que en términos prácticos no manifiestan un riesgo para la vid, dado que niveles de CE menores a $1,5 \mathrm{dS} \mathrm{m}^{-1}$ no afectan los rendimientos (Hoffman y Shannon, 2007). El pH del suelo no varió en los intervalos de profundidad (figura $5 \mathrm{E})$, manteniéndose ligeramente alcalino a alcalino (Navarro y Navarro, 2003). Por último, la vinaza no generó un aporte significativo de $\mathrm{MO}$ al suelo (figura $5 \mathrm{~F}$ ). Esta respuesta al uso de vinaza la reportan Hernández et al. (2008) para el pH y la CE del suelo, y Paula et al. (1999) para K. Considerando que el principal componente de la vinaza es el K, Korndorfer et al. (2010) recomiendan que las dosis de vinaza varíen de acuerdo con el contenido de $\mathrm{K}$ en el suelo, pues cuanto mayor sea su contenido menores serán los requerimientos de vinaza.

Finalmente, frente a la pregunta sobre cuál es el factor que provoca los efectos positivos sobre el desarrollo de las raíces en dosis iguales o menores a $336 \mathrm{~m}^{3} \mathrm{ha}^{-1}$, la respuesta apunta a la acción conjunta del efecto nutricional y la restricción biológica promovida por el residuo orgánico. Un primer factor relevante es el efecto directo del $\mathrm{K}$, el cual incluso puede llegar a tener una acumulación excesiva en el suelo (Hernández et al., 2008). El menor contenido de macronutrientes del suelo $(\mathrm{N}, \mathrm{P}, \mathrm{K}$; figura 5) en el tratamiento de $206 \mathrm{~m}^{3} \mathrm{ha}^{-1}$ no solo responde a una actividad de raíces más intensa (figura 4), sino a una reactivación microbiológica con condiciones físicas adecuadas para la complejación de nutrientes en las fracciones órgano minerales (Chenu y Plante, 2006), lo que impidió tener diferencias en el contenido foliar de nutrientes (tabla 1). Otro factor relevante es el hecho que la vinaza provocó una menor CE respecto del testigo, el que fue fertilizado con fuentes químicas, las cuales son una de las principales causantes de la salinidad de los suelos junto con el agua de riego. Finalmente, es necesario tener presente otros componentes o nutrientes (micronutrientes) que pueden estar presentes en la vinaza y que ameriten ser estudiados para entender de mejor forma la respuesta de plantas de vid ante la aplicación de este tipo de enmiendas.

\section{CONCLUSIONES}

Es recomendable usar hasta $336 \mathrm{~m}^{3}$ ha-1 de vinaza en contenedores, dado que no provocó efectos colaterales negativos en las plantas; por el contrario, promueve un mayor crecimiento de raíces. En campo, hasta $206 \mathrm{~m}^{3}$ ha $^{-1}$ no afectan a corto plazo las condiciones químicas del suelo, el contenido de nutrientes en las plantas, el crecimiento vegetativo evaluado como peso de poda, los rendimientos ni la fecha de cosecha de las vides y promueve un mayor índice de enraizamiento en los primeros $20 \mathrm{~cm}$ de profundidad, reforzando la idea de que el uso adecuado de vinaza del pisco podría ser una alternativa como enmienda orgánica o biofertilizante en los viñedos de la zona árida del desierto de Atacama. 


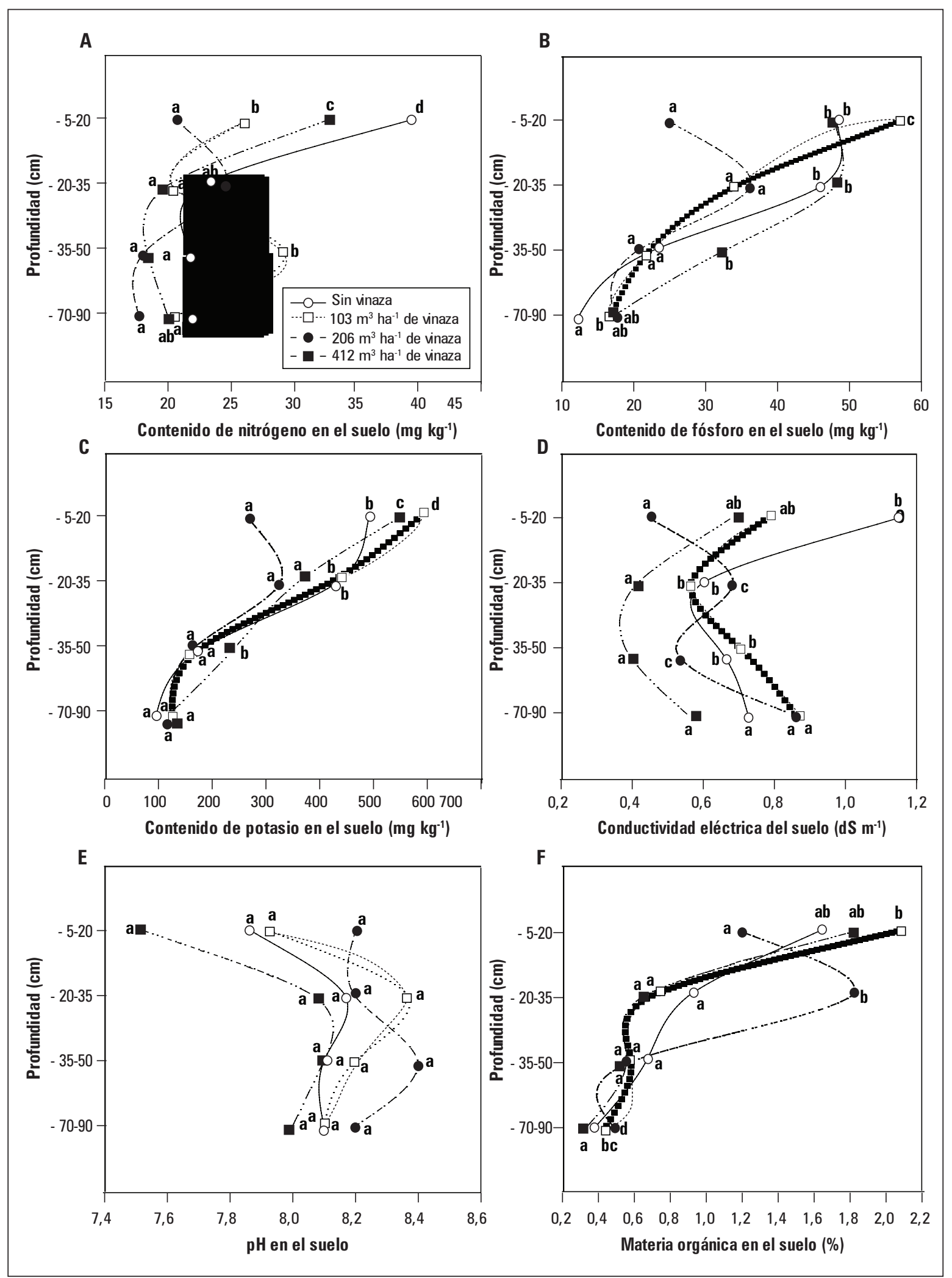

Figura 5. Contenido de A. Nitrógeno, B. Fósforo, C. Potasio, D. Conductividad eléctrica, E. pH y F. Materia orgánica en función de la profundidad del suelo para distintos tratamientos de vinaza. Promedios con letras distintas indican diferencia significativa según la prueba de Tukey $(P \leq 0,05)$. 


\section{REFERENCIAS BIBLIOGRÁFICAS}

Alfaro, R. y J. Alfaro. 1996. Evaluación de la vinaza como fertilizante potásico en la caña de azúcar y su efecto sobre las propiedades químicas de un suelo de Atenas, Alajuela. p. 175. En: Resúmenes X Congreso Nacional Agronómico y II Congreso de Suelos, San José, Costa Rica.

Armengol, J.E., R. Lorenzo y N. Fernández. 2003. Utilización de la vinaza como enmienda orgánica y su influencia en las propiedades químicas de vertisoles y en los rendimientos de la caña de azúcar. Cultivos Trop. 24(3), 67-71.

Bengough, A.G., M.F. Bransby, J. Hans, S.J. Mckenna, T.J. Roberts y A. Valentine. 2006. Root responses to soil physical conditions; growth dynamics from field to cell. J. Exp. Bot. 57(2) 437-447. Doi: 10.1093/jxb/erj003

Böhm, W. 1979. Methods of studying root systems. Springer-Verlag, Berlin.

Cadahía, C. 2005. Fertirrigación: Cultivos hortícolas, frutales y ornamentales. Mundi-Prensa, Madrid.

Callejas, R., A. Bermejillo, E. Kania, G. Reginato, M. Ojer y E. Redonde. 2011. Manejo nutricional. pp. 135-160. En: Ojer, M. (ed). Producción de duraznos para la industria. Federación plan estratégico de durazno industria. Universidad Nacional de Cuyo, Mendoza, Argentina.

Callejas, R., P. Canales y V. García de Cortázar. 2009. Relationship between root growth of 'Thompson Seedless' grapevines and soil temperature. Chilean J. Agric. Res. 69(4), 496-502. Doi: 10.4067/S071858392009000400003

Callejas, R., E. Rojo, C. Benavides y E. Kania. 2012. Crecimiento y distribución de raíces y su relación con el potencial productivo de parrales de vides de mesa. Agrociencia 46, 23-35.

Chenu, C. y A.F. Plante. 2006. Clay-sized organo-mineral complexes in a cultivation chronosequence revisiting the concept of the primary organo-mineral complex. Europ. J. Soil Sci. 57, 596-607. Doi: 10.1111/j.1365-2389.2006.00834.x

CPL (Consejo de Producción Limpia). 2004. Productores de Pisco y Procesadores de Uva Pisquera III y IV Región Chile produce limpio. http://www.produc-
cionlimpia.cl/medios/documentos/APL_Pisco_. pdf; consulta: junio de 2010.

Christensen, L.P. 1984. Nutrient level comparisons of leaf petioles and blades in twenty-six grape cultivars over three years (1979 through 1981). Amer. J. Enol. Vitic. 35(3), 125-133.

Ferguson, L. and S.R. Grattan. 2005. How salinity damages citrus: Osmotic effects and specific ion toxicities. HortTechnol. 15(1), 95-99.

Glenn, D.M. 2000. Physiological effects of incomplete rootzone wetting on plant growth and their implications for irrigations management. HortScience 35, 1041-1043.

Hernández, G., S. Salgado, D. Palma, L. Lagunas, M. Castelán y O. Ruiz. 2008. Vinaza y composta de cachaza como fuente de nutrientes en caña de azúcar en un gleysol mólico de Chiapas, México. Interciencia 33(11), 855-860

Hoffman, G.J. y M.C. Shannon. 2007. Salinity. pp. 131160. En: Lamm, F.R., J.E Ayars y F.S Nakayama (eds.). Microirrigation for crop production. Elsevier, New York, NY.

Honorato, R., H. Silva y C. Bonomelli. 1990. Productividad de parronales de la Región Metropolitana y sus relaciones edáficas. Ciencia Inves. Agr. 17(3), 133-143.

Korndorfer, G.H., A. Nolla y J.M. Ailton. 2010. Manejo, aplicación y valor fertilizante de la vinaza para caña de azúcar y otros cultivos. Tecnicaña (Instituto de Ciencias Agrarias, Universidad Federal de Uberlandia, Uberlândia, MG, Brasil) 24, 23-28.

Mora, X., F. Valverde, R. Parra, Y. Cartagena, S. Alvarado y $\mathrm{R}$. Jaramillo. 2010. Respuesta del cultivo de papa (Solanum tuberosum L.) variedad iniap-estela a la aplicación de vinaza y riego por goteo. p. 11. En: Memorias XII Congreso Ecuatoriano de la Ciencia del Suelo. Santo Domingo, Ecuador.

Mullins, M.G., A. Bouquet y L.E. Williams. 1992. Biology of the grapevine. pp. 37-147. En: Mullins, M.G. (ed). Cambridge University Press, Cambridge, UK.

Navarro, S. y G. Navarro. 2003. Química agrícola. El Suelo y los elementos químicos esenciales para la 
vida vegetal. $2^{\text {a }}$ ed. Ediciones Mundi-Prensa, Barcelona, España.

Obreza, T.A. y A. Schumann. 2010. Keeping water and nutrients in the Florida citrus tree root zone. HortTechnol. 20(1), 67-73.

Paula, M.B., F.S. Holanda, H.A. Mesquita y V.D. Carvalho. 1999. Uso da vinhaca no abacaxizeiro em solo de baixo potencial de producao. Pesq. Agropec. Bras. 34, 1217-1222.

Paula, M.B., V.D. Carvalho y F.D. Nogueira. 1992. Efeitos da vinhaca na producao e qualidade da cebola em solo de baixo potencial de produtividade. Pesq. Agropec. Bras. 27, 389-393.

Pérez, I. y N. Garrido. 2008. Ingeniería química. Tratamiento de aguas residuales. Instituto Cubano de Investigaciones de los Derivados de la Caña de Azúcar (ICIDCA), La Habana. pp. 148-153.

Sadzawka, A., M.A. Carrasco, R. Grez, M. Mora, H. Flores y A. Neaman. 2006. Métodos de análisis recomendados para los suelos de Chile. Serie Actas INIA $\mathrm{N}^{\circ}$ 34. Instituto de Investigaciones Agropecuarias, Santiago.

Santos, M., F. Martin, F. Diánez, F. Carretero, M. García-Alcázar, M. De Cara y J.C. Tello. 2007. Efecto de la aplicación de vinaza de vino como biofertilizante y en el control de enfermedades en el cultivo de pepino. pp .58-66. Memorias VIII Congreso SEAE de Agricultura y Alimentación Ecológica, Universidad de Almería, Almería, España.

Sellés, G., R. Ferreyra, G. Contreras, R. Ahumada, J. Valenzuela y R. Bravo. 2003. Manejo de riego por goteo en uva de mesa cv. Thompson Seedless cultivada en suelos de textura fina. Agric. Téc. 63 (2)180-192.

Servicio Agrícola y Ganadero (SAG). 2006. Guía para proyectos de industrias vitivinícolas que aplican riles al suelo. Gobierno de Chile. https//www.eseia.cl/archivos/20081001.001346.pdf; consulta: agosto de 2010.

Silva, D. y R.C. Beeson Jr. 2011. A large-volume rhizotron for evaluating root growth under naturallike soil moisture conditions. HortScience 46(12), 1677-1682.

Singh, Y., M.S. Gangwar, B.S. Mahapatra, P.C. Srivastava, A. Kulshreshtha, R.B. Manihar, S.K. Mishra, M.K. Dasgupta, D.C. Ghosh, D.K. Majumdar, G.N. Chattopadhyay, P.K. Ganguli, P.S. Munsi y D. Bhattacharya. 1998. Utilization of destilery effluent for crop production and improving soil fertility. Proc. Nat. Symp. Sustain. Agric. in Subhumid Zone. University of Agriculture and Technology, Pantnagar Nainital, India.

Urbano, P. 2002. Fertilización orgánica con vinazas de alcoholera. Vida Rural 155, 50-52.

Valverde, F., Y. Cartagena y R. Parra. 2004. Efecto de la vinaza obtenida de la fabricación de la levadura y aplicada en el agua de riego, sobre el rendimiento de tres cultivos y las características químicas del suelo. p. 21. En: Memorias XII Congreso Ecuatoriano de la Ciencia del Suelo. Santo Domingo, Ecuador.

Van Zyl, J.L. 1988. Response of grapevine to soil water regimes and irrigation systems. pp. 30-43. En: The grapevine root and its environment. Pretoria Viticultural and Oenological Research Institute, Pretoria, Sudáfrica.

Villate, J.L. 2007. Atlas de capacidad sustentadora potencial para el reino templado de Chile. Taller de Licenciatura. Pontificia Universidad Católica de Valparaíso, Valpo, Chile.

Wright, A. y R. Wright. 2004. The Horhizotron ${ }^{\mathrm{TM}}$ a new instrument for measuring root growth. HortTechnol. 14 560-563. 